\title{
Trends in the abundance of demersal species in the southern Aegean Sea*
}

\author{
GEORGE TSERPES* and PANAGIOTA PERISTERAKI \\ Institute of Marine Biology of Crete, P.O. BOX 2214 Iraklion, Greece. E-mail: gtserpes@imbc.gr
}

\begin{abstract}
SUMMARY: The present work identifies trends in the abundance of the main demersal species of the South Aegean Sea utilising catch and effort data derived from an international Mediterranean bottom trawl survey (MEDITS). The experimental surveys were accomplished annually from 1994 to 2000 on 61 pre-defined sampling stations. Selection of stations was based on a depth-stratified random sampling scheme that included five depth strata: 10-50, 50-100, 100-200, 200-500 and 500-800 m. The results suggest the existence of a general increasing trend in the biomass of the demersal species, particularly at the $10-50 \mathrm{~m}$ and 500-800 $\mathrm{m}$ depth strata. Findings are discussed in relation to the fisheries exploitation pattern and productivity of the area.
\end{abstract}

Key words: demersal, abundance, Aegean, Mediterranean.

\section{INTRODUCTION}

Due to the presence of numerous islands, the southern Aegean Sea has some unique topographical and bathymetrical features, which contribute to its zoogeographical distinction from the rest of the Aegean (Papaconstantinou, 1990). Fishing activities are widespread all over the area and groundfish trawling is very common, mainly at depths down to $300 \mathrm{~m}$. As the continental shelf is very narrow, the existing measures that prohibit groundfish trawling within one mile from the coast make a large proportion of the area down to $100 \mathrm{~m}$ unavailable to the bottom trawl fishery. The latter is multi-specific, but similarly to other Mediterranean areas, a small group of species, such as red mullets, hake, shrimps,

\footnotetext{
*Received November 9, 2000. Accepted January 23, 2002
}

etc., account for a large proportion of the total catch and its economic value (Caddy, 1993; Farrugio et al., 1993).

Past researchers have described the demersal fauna of the southern Aegean Sea and have related the species composition of the demersal communities with the depth and substrate characteristics of the area (Bellog, 1948; Ege, 1953; Papaconstantinou and Tsimenides, 1979; Tsimenides et al., 1991; Sinis and Koukouras, 1995; Tserpes et al., 1999). However, due to lack of standardised routine surveys, there is no information on the abundance fluctuation of the main demersal species inhabiting the area (Stergiou et al., 1997a). During the last 15 years, commercial catches show an upward trend, similar to that of the whole Mediterranean (Papaconstantinou and Farrugio, 2000), but it is not known if such an increase reflects abundance 
changes or is simply due to the intensification of fishing activities.

The present work attempts to investigate biomass abundance changes of the main demersal species of the southern Aegean Sea by using a time series of data collected within the framework of an international Mediterranean bottom trawl survey. Moreover, fluctuations in the overall abundance of the different depth zones by faunistic category are examined and the results are discussed in relation to the fishing activities carried out in the area.

\section{MATERIALS AND METHODS}

Within the framework of the "MEDITS" international trawl survey research program, seven annual bottom trawl surveys have been performed from 1994 to 2000 in the southern Aegean Sea (Bertrand et al., 2000, 2002). The surveys, which were mainly aimed at obtaining estimates of abundance indices for a series of target species, were accomplished in summer (June-July), and each of them included sampling at 61 pre-defined stations distributed all over the sampled area (Fig. 1). Selection of stations was based on a depth-strati- fied sampling scheme and the same sampling protocol was used in all cases (Bertrand et al., 2000, 2002). The surveys covered the depth range from $10 \mathrm{~m}$ to $800 \mathrm{~m}$ and the total surface of the surveyed area was estimated to be up to $62,601 \mathrm{~km}^{2}$. The surface of each depth zone was: $10-50 \mathrm{~m}: 4,918 \mathrm{~km}^{2}$, 50-100 m: 4,090 km², 100-200 m: 13,269 km², 200-500 m: $18,100 \mathrm{~km}^{2}, 500-800 \mathrm{~m}: 22,224 \mathrm{~km}^{2}$. The number of the annual hauls fluctuated between 40 and 60 , being close to 60 since 1996 . The fluctuation was due to: (a) the expansion of the sampling scheme introduced in 1996 and (b) the existence of adverse weather conditions that prevented sampling in certain cases.

From the collected data, abundance indices, expressed in terms of $\mathrm{g} / \mathrm{km}^{2}$ of swept area were computed separately for each station, for 14 out of 50 target species included in the MEDITS survey (Bertrand et al., 2000, 2002). The selection of species was based on their: (a) accessibility to commercial bottom trawlers, (b) commercial interest and (c) importance in characterising the demersal fauna of the different depth strata of the southern Aegean Sea (see Tserpes et al. (1999) for details). Out of the 14 selected species, eight were fishes, four cephalopods and two crustaceans (Table 1). For each

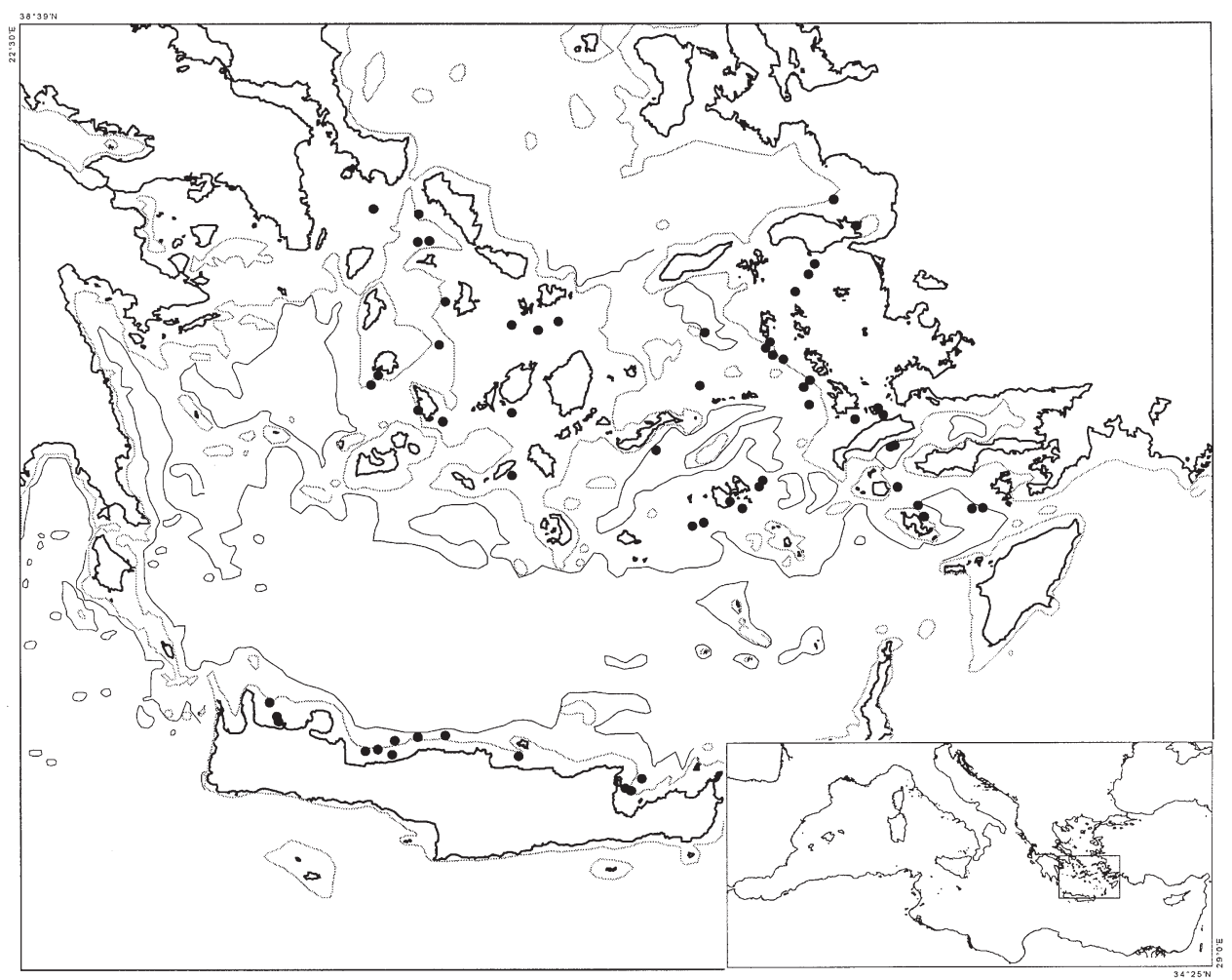

FIG. 1. - Map of the surveyed area indicating the position of the sampling stations (bullets) and the 200 and $500 \mathrm{~m}$ isobaths. The relative position of the surveyed area in the Mediterranean basin is shown on the right bottom sketch of the figure. 
TABLE 1. - Depth zones examined for each species.

\begin{tabular}{|c|c|c|c|c|c|}
\hline Species & $10-50 \mathrm{~m}$ & $50-100 \mathrm{~m}$ & $100-200 \mathrm{~m}$ & $200-500 \mathrm{~m}$ & $500-800 \mathrm{~m}$ \\
\hline Sepia officinalis & + & + & + & & \\
\hline Eledone moschata & + & + & + & & \\
\hline Octopus vulgaris & + & + & + & & \\
\hline Serranus cabrilla & + & + & + & & \\
\hline Pagellus erythrinus & + & + & + & & \\
\hline Mullus barbatus & + & + & + & + & \\
\hline Mullus surmuletus & + & + & + & + & \\
\hline Scyliorhinus canicula & & + & + & + & \\
\hline Eledone cirrhosa & & & + & + & \\
\hline Squalus acanthias & & & + & + & + \\
\hline Merluccius merluccius & & + & + & + & + \\
\hline Parapenaeus longirostris & & & & + & + \\
\hline Phycis blennoides & & & & + & + \\
\hline Aristeomorpha foliacea & & & & & + \\
\hline
\end{tabular}

station, the overall abundance index by faunistic category was also computed taking into account all target species of the survey (50 in total).

In order to examine the effects of year and depth in the abundance indices and identify time trends, in terms of both individual species and faunistic category, catch per unit effort (CPUE) indices were standardised using General Linear Model (GLM) techniques (Gulland 1956; Kimura, 1981). The general model used was of the form:

$$
\ln \left(\mathrm{CPUE}_{\mathrm{ij}}+1\right)=\mu+\mathrm{D}_{\mathrm{i}}+\mathrm{Y}_{\mathrm{j}}+\text { interaction }+\varepsilon_{\mathrm{ij}},
$$

where $\mathrm{CPUE}_{\mathrm{ij}}$ : nominal CPUE at depth stratum $\mathrm{i}$, in year $\mathrm{j}$,

$\mu$ : overall mean,

$\mathrm{D}_{\mathrm{i}}$ : effect of depth stratum $\mathrm{i}$,

$Y_{j}$ : effect of year $j$,

Interaction: two effect interaction, and tributed.

$\varepsilon$ : error term assumed to be normally dis-

The constant 1 was added to all CPUE rates to account for the zero observations. For each species, the analysis was confined to the depth zones where the species was mainly found (Table 1 ). The criterion used was its presence in at least $40 \%$ of the stations of the corresponding depth stratum. The analysis by faunistic category included all depth strata for fish (five in total: 10-50, 50-100, 100200, 200-500, 500-800 m), four strata (all up to $500 \mathrm{~m}$ ) for cephalopods and two for crustaceans (200-500, 500-800 m). To identify time trends, linear trend-lines were plotted on the standardised annual CPUE estimates.

The measure of goodness of fit of the models was the coefficient of determination $\left(\mathrm{R}^{2}\right)$. All statistical inferences were based on the $95 \%$ confidence level.

\section{RESULTS}

\section{Analysis by species}

Analysis of Variance (ANOVA) results for the GLMs fitted to the CPUE rates are presented separately for each species in Tables 2-4. Estimated $\mathrm{R}^{2}$ values indicate that the models explained $12-28 \%$ of the total variation. The annual standardized CPUE indices and the fitted trend-lines are illustrated in Figs. 2-4. For five degrees of freedom (i.e. $n=7$ ) significant trends at the 0.05 significance level were only those having a $\mathrm{R}^{2}$ value higher than 0.57 (corresponding Pearson's correlation coefficient, $r=0.755)$. Significantly increasing abundance trends were found for six species: Mullus barbatus, Mullus surmuletus, Phycis blennoides, Squalus acanthias, Parapenaeus longirostris and Aristeomorpha foliacea. Eledone cirrhosa was the only species that showed a significantly decreasing trend, which however was marginal $\left(\mathrm{R}^{2}\right.$ $=0.571)$. The rest of the species did not show any significant trend. Standardized CPUE indices by species and depth stratum are presented in Fig. 5. The main findings are summarized in Table 5.

\section{Analysis by faunistic category}

The analysis included all target species of the "MEDITS" survey (Bertrand et al., 2000, 2002). ANOVA results are presented separately for each faunistic category in Table 6 . In all cases the fitted GLMs explained about $20 \%$ of the total variation. The overall annual standardized CPUE indices and the fitted trend-lines are illustrated in Fig. 6. The corresponding indices and trend-lines by depth stratum are shown in Figs. 7-9. The main findings are summarized below. 
TABle 2. - ANOVA table for the GLMs fitted to each fish species.SS, Sum of Squares; MS, Mean Square.

\begin{tabular}{|c|c|c|c|c|c|}
\hline $\begin{array}{l}\text { Mullus barbatus } \\
\text { Source }\end{array}$ & $\begin{array}{l}\left(\mathrm{R}^{2}=0.25\right) \\
\mathrm{SS}\end{array}$ & df & MS & $\mathrm{F}$ & Sig. \\
\hline $\begin{array}{l}\text { Intercept } \\
\text { Year } \\
\text { Stratum } \\
\text { Year* Stratum } \\
\text { Error } \\
\text { Corrected Total }\end{array}$ & $\begin{array}{c}4349.6 \\
88.9 \\
1584 \\
156.2 \\
5685.5 \\
7611.8\end{array}$ & $\begin{array}{r}1 \\
6 \\
3 \\
18 \\
291 \\
318\end{array}$ & $\begin{array}{c}4349.6 \\
14.8 \\
528 \\
8.6 \\
19.5\end{array}$ & $\begin{array}{c}222.6 \\
0.7 \\
27 \\
0.4\end{array}$ & $\begin{array}{l}0 \\
0.603 \\
0 \\
0.977\end{array}$ \\
\hline $\begin{array}{l}\text { Mullus surmuletus } \\
\text { Source }\end{array}$ & $\begin{array}{ll}s \quad\left(\mathrm{R}^{2}=0.16\right) \\
\mathrm{SS}\end{array}$ & $\mathrm{df}$ & MS & $\mathrm{F}$ & Sig. \\
\hline $\begin{array}{l}\text { Intercept } \\
\text { Year } \\
\text { Stratum } \\
\text { Year* Stratum } \\
\text { Error } \\
\text { Corrected Total }\end{array}$ & $\begin{array}{r}5079.9 \\
75.3 \\
758.8 \\
202.9 \\
5806.5 \\
6918.2\end{array}$ & $\begin{array}{r}1 \\
6 \\
3 \\
18 \\
291 \\
318\end{array}$ & $\begin{array}{r}5079.9 \\
12.5 \\
252.9 \\
11.2 \\
19.9\end{array}$ & $\begin{array}{r}254.5 \\
0.6 \\
12.6 \\
0.5\end{array}$ & $\begin{array}{l}0 \\
0.707 \\
0 \\
0.923\end{array}$ \\
\hline $\begin{array}{l}\text { Serranus cabrilla } \\
\text { Source }\end{array}$ & $\begin{array}{l}\left(\mathrm{R}^{2}=0.15\right) \\
\mathrm{SS}\end{array}$ & $\mathrm{df}$ & MS & $\mathrm{F}$ & Sig. \\
\hline $\begin{array}{l}\text { Intercept } \\
\text { Year } \\
\text { Stratum } \\
\text { Year* Stratum } \\
\text { Error } \\
\text { Corrected Total }\end{array}$ & $\begin{array}{c}6513.4 \\
55 \\
198.1 \\
114.3 \\
2346 \\
2762.9\end{array}$ & $\begin{array}{r}1 \\
6 \\
2 \\
12 \\
148 \\
168\end{array}$ & $\begin{array}{c}6513.4 \\
9.1 \\
99 \\
9.5 \\
15.8\end{array}$ & $\begin{array}{r}410.9 \\
0.5 \\
6.2 \\
0.6\end{array}$ & $\begin{array}{l}0 \\
0.747 \\
0.002 \\
0.839\end{array}$ \\
\hline $\begin{array}{l}\text { Pagellus erythrint } \\
\text { Source }\end{array}$ & us $\quad \underset{\mathrm{SS}}{\left(\mathrm{R}^{2}=0.26\right)}$ & $\mathrm{df}$ & MS & $\mathrm{F}$ & Sig. \\
\hline $\begin{array}{l}\text { Intercept } \\
\text { Year } \\
\text { Stratum } \\
\text { Year* Stratum } \\
\text { Error } \\
\text { Corrected Total }\end{array}$ & $\begin{array}{r}4033.9 \\
101.7 \\
1006.5 \\
72.9 \\
3304.5 \\
4460.5\end{array}$ & $\begin{array}{r}1 \\
6 \\
2 \\
12 \\
148 \\
168\end{array}$ & $\begin{array}{c}4033.9 \\
16.9 \\
503.2 \\
6 \\
22.3\end{array}$ & $\begin{array}{r}180.6 \\
0.7 \\
22.5 \\
0.2\end{array}$ & $\begin{array}{l}0 \\
0.603 \\
0 \\
0.993\end{array}$ \\
\hline $\begin{array}{l}\text { Scyliorhinus canic } \\
\text { Source }\end{array}$ & $\begin{array}{l}\operatorname{cula}\left(\mathrm{R}^{2}=0.25\right) \\
\mathrm{SS}\end{array}$ & $\mathrm{df}$ & MS & F & Sig. \\
\hline $\begin{array}{l}\text { Intercept } \\
\text { Stratum } \\
\text { Year } \\
\text { Stratum * Year } \\
\text { Error } \\
\text { Corrected Total }\end{array}$ & $\begin{array}{r}8647.8 \\
1566.1 \\
127.2 \\
285.5 \\
6445.4 \\
8550.3\end{array}$ & $\begin{array}{r}1 \\
3 \\
6 \\
17 \\
318 \\
344\end{array}$ & $\begin{array}{c}8647.8 \\
522 \\
21.2 \\
16.7 \\
20.2\end{array}$ & $\begin{array}{c}426.6 \\
25.7 \\
1 \\
0.8\end{array}$ & $\begin{array}{l}0 \\
0 \\
0.395 \\
0.659\end{array}$ \\
\hline $\begin{array}{l}\text { Squalus acanthias } \\
\text { Source }\end{array}$ & $\begin{array}{l}\left(\mathrm{R}^{2}=0.27\right) \\
\mathrm{SS}\end{array}$ & $\mathrm{df}$ & MS & $\mathrm{F}$ & Sig. \\
\hline $\begin{array}{l}\text { Intercept } \\
\text { Stratum } \\
\text { Year } \\
\text { Stratum * Year } \\
\text { Error } \\
\text { Corrected Total }\end{array}$ & $\begin{array}{r}4074.6 \\
1417.3 \\
599.1 \\
135.6 \\
6748.1 \\
9218.7\end{array}$ & $\begin{array}{r}1 \\
2 \\
6 \\
12 \\
273 \\
293\end{array}$ & $\begin{array}{r}4074.6 \\
708.6 \\
99.8 \\
11.3 \\
24.7\end{array}$ & $\begin{array}{c}164.8 \\
28.6 \\
4 \\
0.4\end{array}$ & $\begin{array}{l}0 \\
0 \\
0.001 \\
0.938\end{array}$ \\
\hline $\begin{array}{l}\text { Merluccius merlu } \\
\text { Source }\end{array}$ & 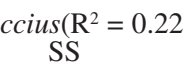 & 2) $\mathrm{df}$ & MS & $\mathrm{F}$ & Sig. \\
\hline $\begin{array}{l}\text { Intercept } \\
\text { Year } \\
\text { Stratum } \\
\text { Year* Stratum } \\
\text { Error } \\
\text { Corrected Total }\end{array}$ & $\begin{array}{c}15998.8 \\
269.8 \\
379 \\
731.8 \\
4600.6 \\
5888.1\end{array}$ & $\begin{array}{r}1 \\
6 \\
3 \\
18 \\
326 \\
353\end{array}$ & $\begin{array}{r}15998.8 \\
44.9 \\
126.3 \\
40.6 \\
14.1\end{array}$ & $\begin{array}{r}1133.6 \\
3.1 \\
8.9 \\
2.8\end{array}$ & $\begin{array}{l}0 \\
0.005 \\
0 \\
0\end{array}$ \\
\hline $\begin{array}{l}\text { Phycis blennoides } \\
\text { Source }\end{array}$ & $\begin{array}{l}\left(\mathrm{R}^{2}=0.12\right) \\
\mathrm{SS}\end{array}$ & $\mathrm{df}$ & MS & $\mathrm{F}$ & Sig. \\
\hline $\begin{array}{l}\text { Intercept } \\
\text { Stratum } \\
\text { Year } \\
\text { Stratum * Year } \\
\text { Error } \\
\text { Corrected Total }\end{array}$ & $\begin{array}{r}4340.5 \\
11.2 \\
197.3 \\
223.6 \\
2931.7 \\
3331.3\end{array}$ & $\begin{array}{r}1 \\
1 \\
6 \\
6 \\
193 \\
206\end{array}$ & $\begin{array}{r}4340.5 \\
11.2 \\
32.8 \\
37.2 \\
15.1\end{array}$ & $\begin{array}{r}285.7 \\
0.7 \\
2.1 \\
2.4\end{array}$ & $\begin{array}{l}0 \\
0.391 \\
0.048 \\
0.026\end{array}$ \\
\hline
\end{tabular}

TABLE 3. - ANOVA table for the GLMs fitted to each cephalopod species. SS, Sum of Squares; MS, Mean Square.

\begin{tabular}{|c|c|c|c|c|c|}
\hline \multirow{2}{*}{$\begin{array}{l}\text { Sepia officinalis } \\
\text { Source }\end{array}$} & \multicolumn{2}{|c|}{$\left(\mathrm{R}^{2}=0.17\right)$} & \multirow[b]{2}{*}{ MS } & \multirow[b]{2}{*}{ F } & \multirow[b]{2}{*}{ Sig. } \\
\hline & SS & df & & & \\
\hline Intercept & 1739.8 & 1 & 1739.8 & 85.7 & 0 \\
\hline Stratum & 329.8 & 2 & 164.9 & 8.1 & 0 \\
\hline Year & 99.2 & 6 & 16.5 & 0.8 & 0.56 \\
\hline Stratum * Year & 107.4 & 12 & 8.9 & 0.4 & 0.944 \\
\hline Error & 3002.2 & 148 & 20.2 & & \\
\hline Corrected Total & 3633 & 168 & & & \\
\hline Octopus vulgaris & \multicolumn{2}{|c|}{$\left(\mathrm{R}^{2}=0.20\right)$} & & & \\
\hline Source & SS & $\mathrm{df}$ & MS & $\mathrm{F}$ & Sig. \\
\hline Intercept & 2981.2 & 1 & 2981.2 & 133 & 0 \\
\hline Stratum & 410.8 & 2 & 205.4 & 9.1 & 0 \\
\hline Year & 196.9 & 6 & 32.8 & 1.4 & 0.194 \\
\hline Stratum * Year & 179.4 & 12 & 14.9 & 0.6 & 0.78 \\
\hline Error & 3361.7 & 150 & 22.4 & & \\
\hline Corrected Total & 4186.2 & 170 & & & \\
\hline Eledone moschata & \multicolumn{2}{|c|}{$\left(\mathrm{R}^{2}=0.25\right)$} & & & \\
\hline Source & $\mathrm{SS}$ & df & MS & $\mathrm{F}$ & Sig. \\
\hline Intercept & 5060 & 1 & 5060 & 267.4 & 0 \\
\hline Year & 193.6 & 6 & 32.2 & 1.7 & 0.123 \\
\hline Stratum & 468.1 & 2 & 234 & 12.3 & 0 \\
\hline Year* Stratum & 125.5 & 12 & 10.4 & 0.5 & 0.876 \\
\hline Error & 2799.9 & 148 & 18.9 & & \\
\hline Corrected Total & 3727.1 & 168 & & & \\
\hline Eledone cirrhosa & \multicolumn{2}{|c|}{$\left(\mathrm{R}^{2}=0.28\right)$} & & & \\
\hline Source & $\mathrm{SS}$ & $\mathrm{df}$ & MS & $\mathrm{F}$ & Sig. \\
\hline Intercept & 1972.9 & 1 & 1972.9 & 140.1 & 0 \\
\hline Year & 824.2 & 6 & 137.3 & 9.7 & 0 \\
\hline Stratum & 118.3 & 1 & 118.3 & 8.4 & 0.004 \\
\hline Year * Stratum & 314.1 & 6 & 52.3 & 3.7 & 0.002 \\
\hline Error & 2842.6 & 202 & 14 & & \\
\hline Corrected Total & 3968.4 & 215 & & & \\
\hline
\end{tabular}

TABLE 4. - ANOVA table for the GLMs fitted to each crustacean species. SS, Sum of Squares; MS, Mean Square.

\begin{tabular}{|c|c|c|c|c|c|}
\hline \multicolumn{6}{|c|}{ Parapenaeus longirostris $\left(\mathrm{R}^{2}=0.17\right)$} \\
\hline Source & SS & df & MS & $\mathrm{F}$ & Sig. \\
\hline Intercept & 4618.1 & 1 & 4618.1 & 262.6 & 0 \\
\hline Year & 456.1 & 6 & 76 & 4.3 & 0 \\
\hline Stratum & 108.5 & 1 & 108.5 & 6.1 & 0.014 \\
\hline Year* Stratum & 182.2 & 6 & 30.3 & 1.7 & 0.116 \\
\hline Error & 3340.5 & 190 & 17.5 & & \\
\hline Corrected Total & 4047.1 & 203 & & & \\
\hline \multicolumn{6}{|c|}{ Aristeomorpha foliacea $\quad\left(\mathrm{R}^{2}=0.24\right)$} \\
\hline Source & SS & df & MS & $\mathrm{F}$ & Sig. \\
\hline Intercept & 1456.8 & 1 & 1456.8 & 82.6 & 0 \\
\hline Year & 239.2 & 5 & 47.8 & 2.7 & 0.032 \\
\hline Error & 757.9 & 43 & 17.6 & & \\
\hline Corrected Total & 997.2 & 48 & & & \\
\hline
\end{tabular}

Fish

ANOVA indicated that differences among years and depth strata were significant (Table 6). Annual standardized CPUE showed a significantly increasing trend with a $\mathrm{R}^{2}$ value equal to 0.73 (Fig. 6). 

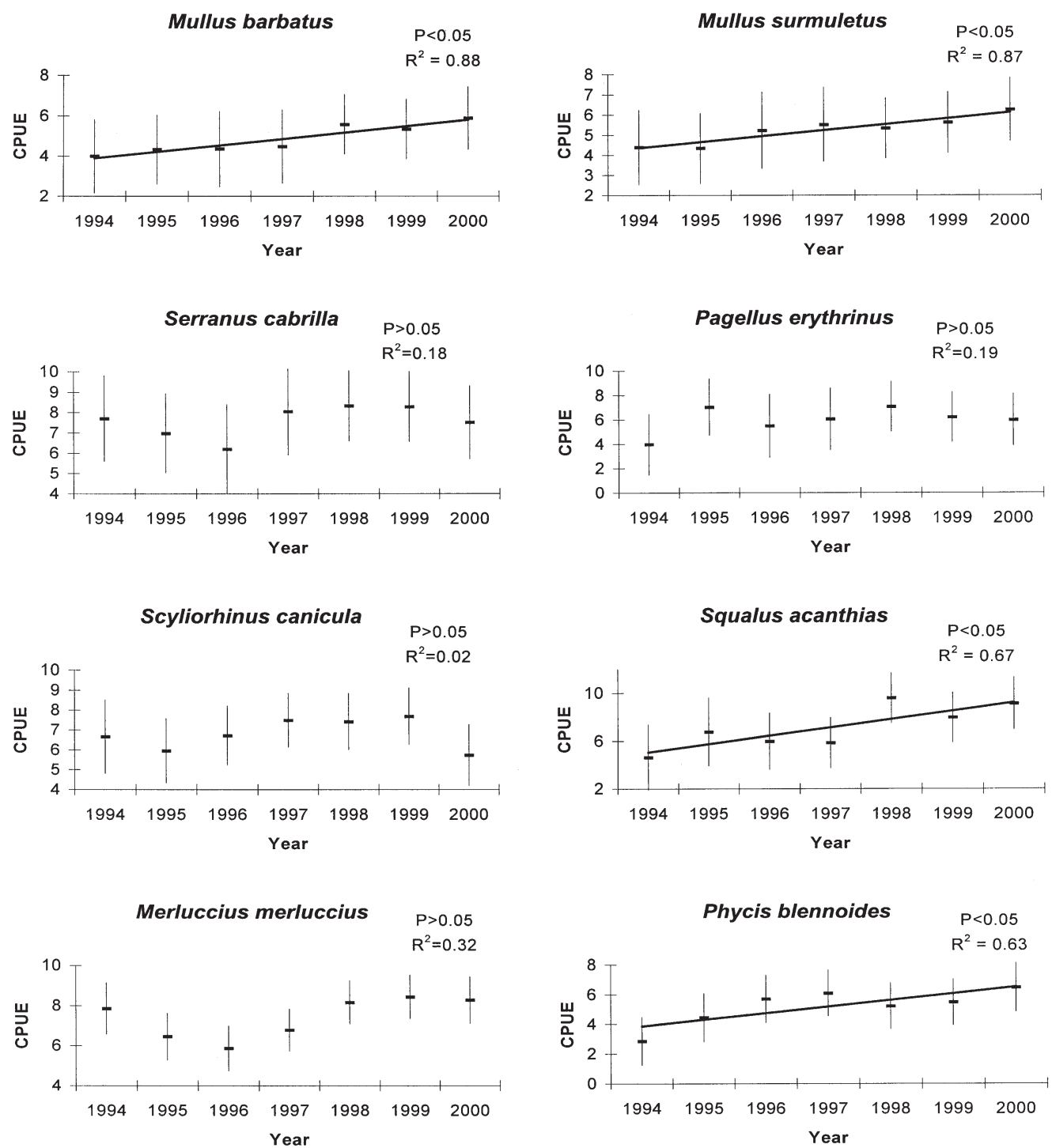

FIG. 2. - Standardised CPUE estimates expressed in $\ln \left(\mathrm{g} / \mathrm{km}^{2}\right)$ by year and fish species. Vertical lines indicate the $95 \%$ confidence intervals of the corresponding estimates. Trend-lines indicate the presence of significant trends.
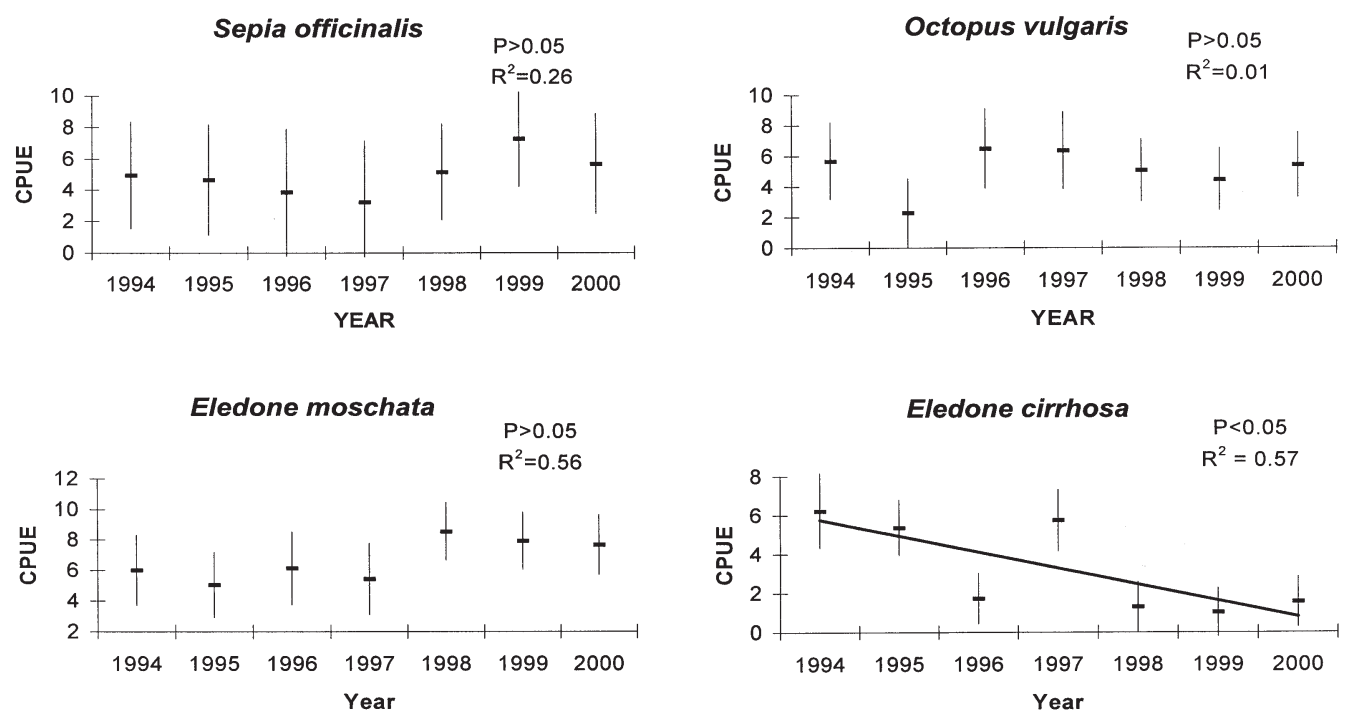

FIG. 3. - Standardised CPUE estimates expressed in $\ln \left(\mathrm{g} / \mathrm{km}^{2}\right)$ by year and cephalopod species. Vertical lines indicate the $95 \%$ confidence intervals of the corresponding estimates. Trend-lines indicate the presence of significant trends. 

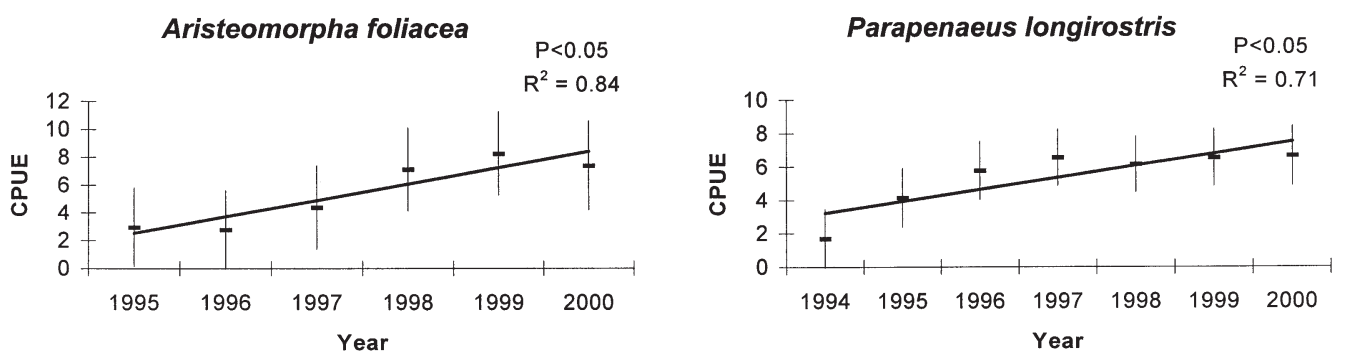

FIG. 4. - Standardised CPUE estimates expressed in $\ln \left(\mathrm{g} / \mathrm{km}^{2}\right)$ by year and crustacean species. Vertical lines indicate the $95 \%$ confidence intervals of the corresponding estimates. Trend-lines indicate the presence of significant trends.
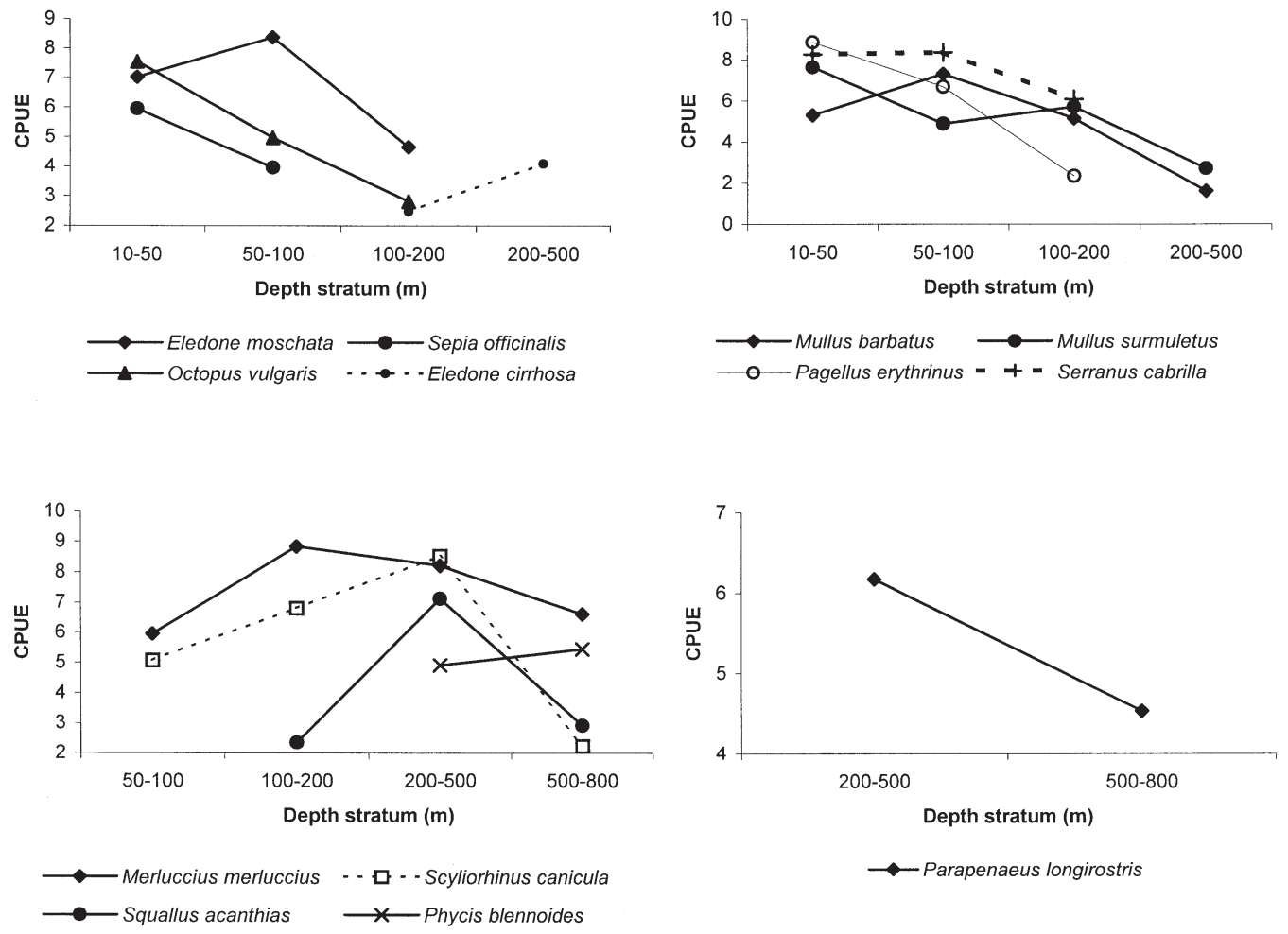

FIG. 5. - Standardized overall CPUE estimates expressed in $\ln \left(\mathrm{g} / \mathrm{km}^{2}\right)$ by species and depth stratum.

TABLE 5. - Summarised results by species of the fitted GLMs and linear trend-lines (see text for details). Tick-marks indicate the presence of significant differences.

\begin{tabular}{|c|c|c|c|c|c|}
\hline & \multicolumn{3}{|c|}{ ANOVA results } & \multirow[b]{2}{*}{$\begin{array}{l}\text { Depth strata of } \\
\text { higher abundance }\end{array}$} & \multirow[b]{2}{*}{$\begin{array}{l}\text { Significance of } \\
\text { abundance trends }\end{array}$} \\
\hline & Stratum & Year & $\begin{array}{l}\text { Stratum-year } \\
\text { interaction }\end{array}$ & & \\
\hline Merluccius merluccius & $\sqrt{ }$ & $\sqrt{ }$ & $\sqrt{ }$ & $100-200,200-500$ & No trend $\left(\mathrm{R}^{2}=0.32, \mathrm{P}>0.05\right)$ \\
\hline Mullus barbatus & $\sqrt{ }$ & & & $50-100$ & Increase $\left(\mathrm{R}^{2}=0.88, \mathrm{P}<0.05\right)$ \\
\hline Mullus surmuletus & $\sqrt{ }$ & & & $10-50$ & Increase $\left(\mathrm{R}^{2}=0.87, \mathrm{P}<0.05\right)$ \\
\hline Pagellus erythrinus & $\sqrt{ }$ & & & $10-50$ & No trend $\left(\mathrm{R}^{2}=0.19, \mathrm{P}>0.05\right)$ \\
\hline Phycis blennoides & & $\sqrt{ }$ & $\sqrt{ }$ & $500-800$ & Increase $\left(\mathrm{R}^{2}=0.63, \mathrm{P}<0.05\right)$ \\
\hline Scyliorhinus canicula & $\sqrt{ }$ & & & $200-500$ & No trend $\left(R^{2}=0.02, P>0.05\right)$ \\
\hline Serranus cabrilla & $\sqrt{ }$ & & & $50-100$ & No trend $\left(\mathrm{R}^{2}=0.18, \mathrm{P}>0.05\right)$ \\
\hline Squalus acanthias & $\sqrt{ }$ & $\sqrt{ }$ & & $200-500$ & Increase $\left(\mathrm{R}^{2}=0.67, \mathrm{P}<0.05\right)$ \\
\hline Eledone cirrhosa & $\sqrt{ }$ & $\sqrt{ }$ & $\sqrt{ }$ & $200-500$ & Decrease $\left(\mathrm{R}^{2}=0.57, \mathrm{P}<0.05\right)$ \\
\hline Eledone moschata & $\sqrt{ }$ & & & $10-50$ & No trend $\left(\mathrm{R}^{2}=0.56, \mathrm{P}>0.05\right)$ \\
\hline Octopus vulgaris & $\sqrt{ }$ & & & $10-50$ & No trend $\left(R^{2}=0.01, P>0.05\right)$ \\
\hline Sepia officinalis & $\sqrt{ }$ & & & $10-50$ & No trend $\left(R^{2}=0.26, P>0.05\right)$ \\
\hline Parapenaeus longirostris & $\sqrt{ }$ & & & $200-500$ & Increase $\left(\mathrm{R}^{2}=0.71, \mathrm{P}<0.05\right)$ \\
\hline Aristeomorpha foliacea & $\sqrt{ }$ & & & $500-800$ & Increase $\left(\mathrm{R}^{2}=0.84, \mathrm{P}<0.05\right)$ \\
\hline
\end{tabular}


TABLE 6. - ANOVA table for the GLMs fitted to each faunistic category. SS, Sum of Squares; MS, Mean Square.

\begin{tabular}{|c|c|c|c|c|c|}
\hline Fish & \multicolumn{2}{|c|}{$\left(\mathrm{R}^{2}=0.22\right)$} & & & \\
\hline Source & $\mathrm{SS}$ & df & MS & $\mathrm{F}$ & Sig. \\
\hline Intercept & 34725.5 & 1 & 34725.5 & 6678 & 0 \\
\hline Stratum & 144 & 4 & 36 & 6.9 & 0 \\
\hline Year & 168.1 & 6 & 28 & 5.3 & 0 \\
\hline Stratum * Year & 114.9 & 24 & 4.7 & 0.9 & 0.573 \\
\hline Error & 1778.3 & 342 & 5.2 & & \\
\hline Corrected Total & 2279.7 & 376 & & & \\
\hline Cephalopods & \multicolumn{2}{|c|}{$\left(\mathrm{R}^{2}=0.22\right)$} & & & \\
\hline Source & SS & $\mathrm{df}$ & MS & $\mathrm{F}$ & Sig. \\
\hline Intercept & 18262.2 & 1 & 18262.2 & 2846 & 0 \\
\hline Stratum & 118 & 3 & 39.3 & 6.1 & 0 \\
\hline Year & 112.7 & 6 & 18.7 & 2.9 & 0.009 \\
\hline Stratum * Year & 161.6 & 18 & 8.9 & 1.4 & 0.13 \\
\hline Error & 1867 & 291 & 6.4 & & \\
\hline Corrected Total & 2399.1 & 318 & & & \\
\hline Crustaceans & \multicolumn{2}{|c|}{$\left(\mathrm{R}^{2}=0.21\right)$} & & & \\
\hline Source & SS & df & MS & $\mathrm{F}$ & Sig. \\
\hline Intercept & 6647.5 & 1 & 6647.5 & 376.4 & 0 \\
\hline Stratum & 40.9 & 1 & 40.9 & 2.3 & 0.129 \\
\hline Year & 671.6 & 6 & 111.9 & 6.3 & 0 \\
\hline Stratum * Year & 409.6 & 6 & 68.2 & 3.8 & 0.001 \\
\hline Error & 3408 & 193 & 17.6 & & \\
\hline Corrected Total & 4318.2 & 206 & & & \\
\hline
\end{tabular}
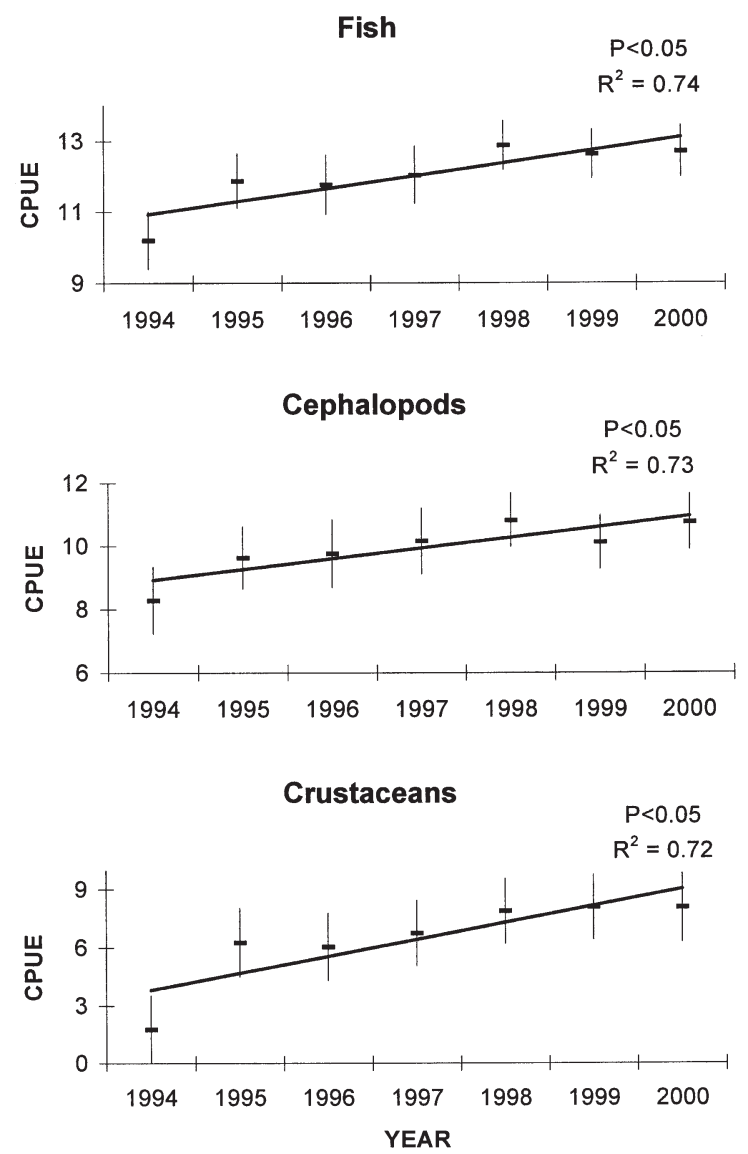

FIG. 6. - Standardised CPUE estimates expressed in $\mathrm{ln}\left(\mathrm{g} / \mathrm{km}^{2}\right)$ by year and faunistic category for all MEDITS species. Vertical lines indicate the $95 \%$ confidence intervals of the corresponding estimates. Trend-lines indicate the presence of significant trends.
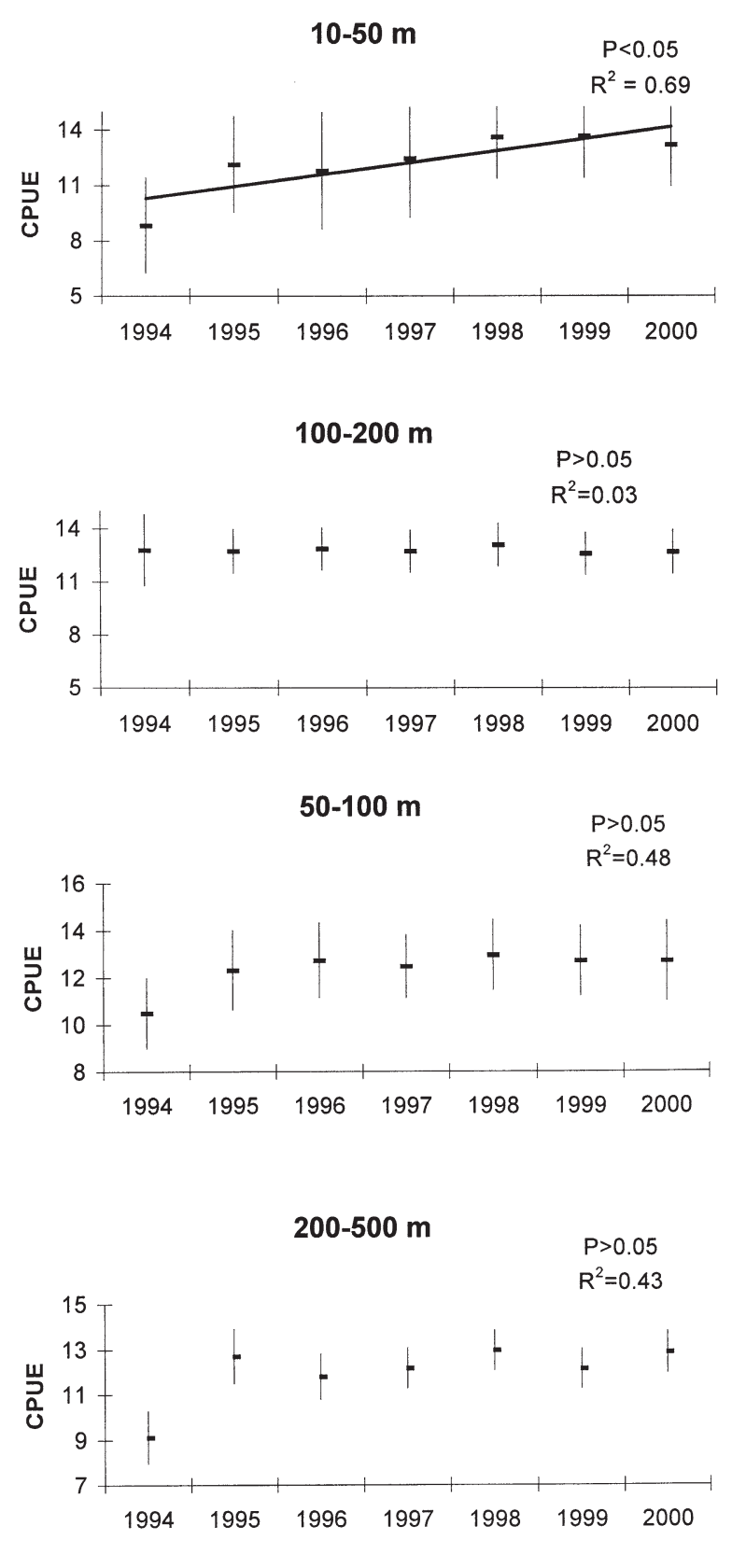

$500-800 \mathrm{~m}$

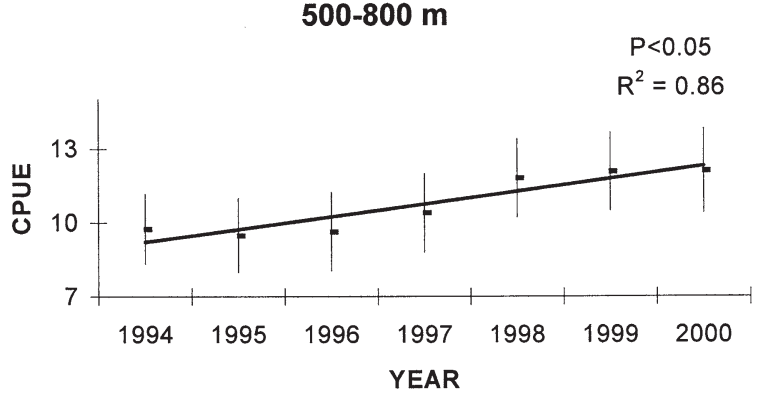

FIG. 7. - Standardised CPUE estimates expressed in $\ln \left(\mathrm{g} / \mathrm{km}^{2}\right)$ by year and depth stratum for all MEDITS fish species. Vertical lines indicate the $95 \%$ confidence intervals of the corresponding estimates. Trend-lines indicate the presence of significant trends. 

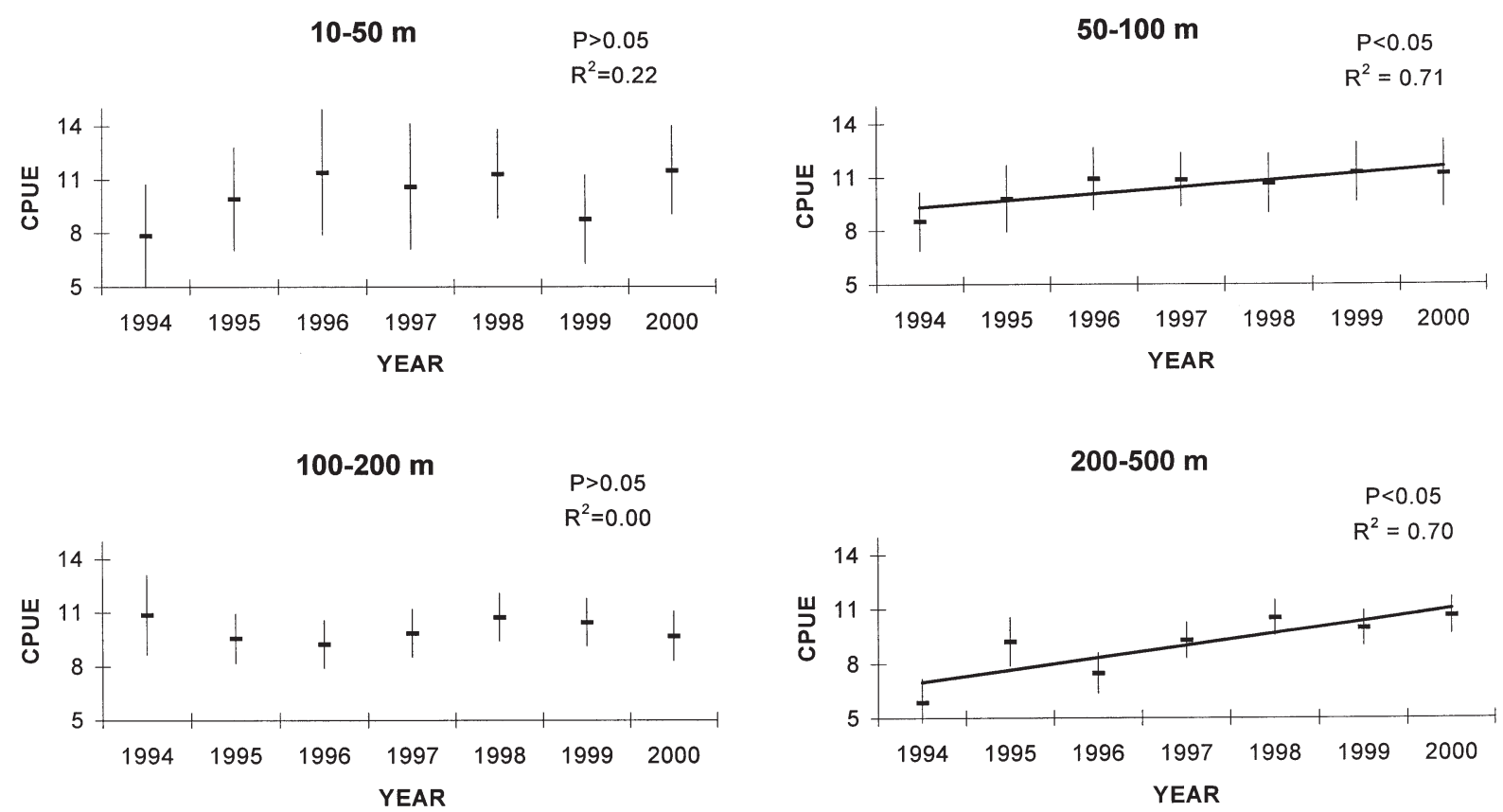

FIG. 8. - Standardised CPUE estimates expressed in $\ln \left(\mathrm{g} / \mathrm{km}^{2}\right)$ by year and depth stratum for all MEDITS cephalopod species. Vertical lines indicate the $95 \%$ confidence intervals of the corresponding estimates. Trend-lines indicate the presence of significant trends.

Analysis by depth stratum demonstrated that the annual standardized CPUE increased significantly with time in the 10-50 and 500-800 m depth zones $\left(\mathrm{R}^{2}=0.68\right.$ and 0.85 , respectively, $\left.\mathrm{P}<0.05\right)$ while no significant trend was detected in the 100-200 and 200-500 m depth zones $\left(\mathrm{R}^{2}=0.03\right.$ and 0.43 , respectively, P>0.05) (Fig. 7).

\section{Cephalopods}

ANOVA indicated that differences among years and depth strata were significant (Table 6). Annual standardised CPUE increased significantly with time $\left(\mathrm{R}^{2}=0.72, \mathrm{P}<0.05\right)$ (Fig. 6). Analysis by depth stratum demonstrated that the annual standardised CPUE had a significantly increasing trend in the 50-100 and 200-500 $\mathrm{m}$ depth zones $\left(\mathrm{R}^{2}=\right.$
0.71 and 0.70 , respectively, $\mathrm{P}<0.05$ ) (Fig. 8). No significant trend was detected in the 10-500 and $100-200 \mathrm{~m}$ depth zones $\left(\mathrm{R}^{2}=0.22\right.$ and 0.00 , respectively, $\mathrm{P}>0.05$ ).

\section{Crustaceans}

ANOVA indicated that differences among years, as well as the year-depth stratum interaction were significant (Table 6). Annual standardised overall CPUE showed a significantly increasing trend $\left(\mathrm{R}^{2}=\right.$ $0.71, \mathrm{P}<0.05$ ) (Fig. 6). Analysis by depth stratum demonstrated that the annual standardised CPUE increased significantly with time for the 500-800 m depth zone $\left(\mathrm{R}^{2}=0.84, \mathrm{P}<0.05\right)$. No significant trend was detected in the $200-500 \mathrm{~m}$ depth zone $\left(\mathrm{R}^{2}=\right.$ 0.06, P>0.05) (Fig. 9).
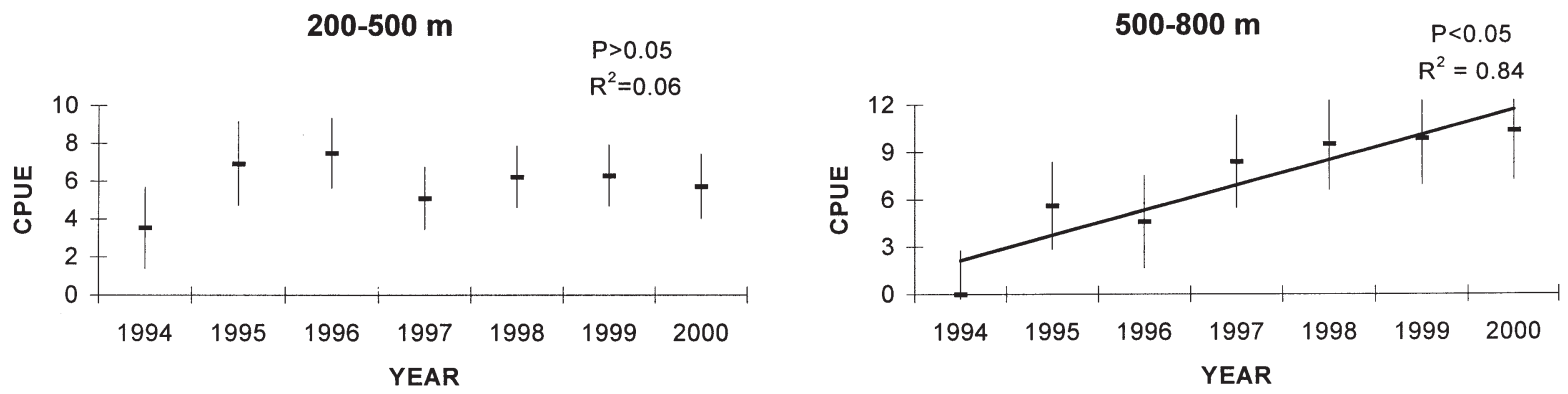

FIG. 9. - Standardised CPUE estimates expressed in $\ln \left(\mathrm{g} / \mathrm{km}^{2}\right)$ by year and depth stratum for all MEDITS crustacean species. Vertical lines indicate the $95 \%$ confidence intervals of the corresponding estimates. Trend-lines indicate the presence of significant trends. 


\section{DISCUSSION}

The present study is the first attempt to examine biomass fluctuations of demersal species in the Aegean Sea. Other authors have analysed time series of catches of the Greek fishing fleets (Stergiou and Christou, 1996; Stergiou et al., 1997b) but, due to lack of appropriate effort data, no comments could be made on aspects related to stock abundance. In the present case the use of generalised linear modelling permitted spatio-temporal comparisons and identification of time-trends. Although the available time-series of data was short for a full time series analysis, the results gave valuable information regarding the fluctuation of the demersal resources in the southern Aegean Sea. Similar techniques have been extensively used in the past for analysing catch-effort data, mainly in the Atlantic and Pacific areas (Gulland, 1956; Gavaris, 1980; Kimura, 1981; Punt et al., 2000). In the case of the Mediterranean Sea, application of general linear modelling in catch-effort data from commercial bottom trawlers provided useful information on the fluctuation of hake stocks in the western Mediterranean (Goñi et al., 1999). Estimates of experimental surveys like "MEDITS" may be affected by the limited duration of the surveys but they are independent of factors such as fisheries behaviour that could bias those estimates based on commercial data.

Our results suggest the existence of a general increasing trend in the biomass of the demersal fauna of the southern Aegean Sea. The eastern Mediterranean was formerly considered as an area of low productivity but it seems that the situation has changed in recent years and there are indications of increased eutrophication in several areas including the Aegean Sea (Bethoux et al. 1998; Caddy et al., 1995; Papaconstantinou and Farrugio, 2000; Theocharis and Lascaratos, 2000). It is possible that the increased productivity has favoured recruitment of several species resulting in a total biomass increase.

An increasing trend in the commercial landings over the last 15 years can also be observed from the records of the National Statistical Service of Greece (Stergiou et al., 1997b). Pauly et al. (1998), who analysed historical FAO fish landings statistics, pointed out that the increasing trend of the world landings during the last 45 years is accompanied by a general decrease of their mean trophic level due to a gradual transition from long-lived, high trophic level, piscivorous bottom fish toward short-lived, low trophic level invertebrates and planktivorous fish. Stergiou and Koulouris (2000) based on the catch data of the National Statistical Service of Greece concluded that this argument is generally justified in the Aegean Sea. The above approach, known as the "fishing down the food web" theory, hints that the abundance of species belonging to high trophic levels (e.g. demersals) should decline with time. The present findings do not support this theory that, however, has been criticised by Caddy et al. (1998) as oversimplifying a complicate situation.

It is noticeable that the stronger increasing trends have been observed in the 10-50 and the $500-800 \mathrm{~m}$ depth strata. Both strata undergo less fishing pressure than the remaining depth ranges due to the established fishing limitations and the behaviour of the fishermen. Thus, both strata are very little or not at all exploited by trawlers.

The existing measures that do not allow groundfish trawling within one mile from the coast make the 10-50 m depth stratum in an area with a narrow continental shelf, such as the southern Aegean, practically unavailable to the trawlers. In addition, as traditionally happens in most Mediterranean areas, fishing activities are focused on the continental shelf, leaving the slope, and particularly areas deeper than $500 \mathrm{~m}$, less disturbed (Caddy, 1993). The above fishing pattern may, at least partially, explain the fact that the highly exploited 100-200 m depth stratum did not show any increasing trend as did the other strata.

Regarding trends at the species level, species that mainly inhabit the slope showed significantly increasing trends. This was particularly true in the case of the red shrimp A. foliacea, which inhabits the deeper part of the slope $(>500 \mathrm{~m})$. Moreover, two coastal species, such as the red and striped mullets (Mullus barbatus and M. surmuletus), which are among the main target species of several fisheries, showed significantly increasing trends. Based on the aforementioned indications on increased productivity, it can be speculated that such a situation has supported recruitment and growth rates that compensate for the high fishing pressure imposed on these species in coastal areas. It is also expected that the closed season for bottom trawlers operating in the Greek seas from June to the end of September, favours the survival of recruits. Finally, the ability of some Mullus species, such as M. barbatus, to delay their settlement until they reach suitable substrates (Kendall et al., 1984), may also further contribute to a successful recruitment. 
Eledone cirrhosa was the only species that showed decreasing trends while E. moschata, which is a more coastal species than its congeneric, showed an increasing, however not significant, trend. This finding cannot be easily explained, but since both are short-lived species (Boyle, 1983; Mangold, 1983 ) it is very likely that their biomass fluctuations follow their recruitment pattern. Consequently, it seems that in the area there were conditions that favoured recruitment of E. moschata and undermined that of E. cirrhosa. This is not unlikely as the species differ in their reproduction period (Mangold et al., 1971; Mangold, 1983).

As the surveys have been carried out at the end, or just after the reproduction period of several species, it was thought that findings could be biased in some cases due to the irregular presence of recruits in the samples. For this reason, the use of biomass indices was preferred over the numeric ones, since they are expected to be less sensitive to spatio-temporal fluctuations due to the presence of recruits.

\section{REFERENCES}

Bellog, G. - 1948. Inventory of the fisheries resources of the Greek waters. Appendix B: Catalogue of the resources of Greek waters, Pisces :64 p. (mimeo).

Bertrand, J.A., L. Gil de Sola, C. Papaconstantinou, G. Relini and A. Souplet. -2000 . An international bottom trawl survey in the Mediterranean: the MEDITS programme. In: J.A. Bertrand and G. Relini (eds.), Demersal resources in the Mediterranean, Proceedings of the Symposium held in Pisa, 18-21 March 1998, Actes de Colloques 26, pp. 76-93. IFREMER, Plouzané.

Bertrand, J., L. Gil de Sola, C. Papaconstantinou, G. Relini and A. Souplet. - 2002. The general specifications of the MediTs surveys. Sci. Mar., 66 (Suppl. 2): 9-17.

Bethoux, J.P., P. Morin, C. Chaumery, O. Connan, B. Gentili and D. Ruiz-Pino. - 1998. Nutrients in the Mediterranean Sea, mass balance and statistical analysis of concentrations with respect to environmental change. Mar. Chem., 63: 155-169.

Boyle, P.R. - 1983. Eledone cirrhosa. In: P.R. Boyle (ed.), Cephalopod Life Cycles, Vol. 1, pp. 365-386. Academic Press, London.

Caddy, J.F. - 1993. Some future perspectives for assessment and management of Mediterranean fisheries. Sci. Mar., 57: 121-130.

Caddy, J.F., J. Csirke, S.M. Garcia and R.J.R. Grainger. - 1998. How pervasive is "fishing down marine food webs"? Science, 282: 1383-1384.

Caddy J.F., R. Refk and T. Do Chi. - 1995. Productivity estimates for the Mediterranean: evidence of accelerating ecological change. Ocean Coast. Manag., 26: 1-18.
Ege, V. - 1953. Paralepidae I (Paralepsis and Lestidium). Taxonomy, ontogeny, phylogeny, and distribution. Dana Rep., 40 : $1-185$.

Farrugio, H., P. Oliver and F. Biagi. - 1993. An overview of the history, knowledge, recent and future research trends in Mediterranean fisheries. Sci. Mar., 57: 105-119.

Gavaris, S. - 1980. Use of a multiplicative model to estimate catch rate and effort from commercial data. Can. J. Fish. Aquat. Sci., 37: 2272-2275.

Goñi, R., F. Alvarez and S. Adlerstein. - 1999. Application of generalized linear modelling to catch rate analysis of Western Mediterranean fisheries: the Castellón trawl fleet as a case study. Fish. Res., 42: 291-302.

Gulland, J.A. - 1956. On the fishing effort in English demersal fisheries. Fish. Invest. London series 2(20): 1-41.

Kendall, A.W., E.H. Ahlstrom and H.G. Moser. - 1984. Early life history stages of fishes and their characters. In: H.G. Moser, W.J. Richards, D.M. Cohen, M.P. Fahay, A.W. Kendall, Jr., and S.L. Richardson (eds.) Ontogeny and Systematics of Fishes, pp. 11-22. Am. Soc. Ichthyol. Herpetol., Spec. Publ. No 1. Allen Press Inc, Lawrence.

Kimura, D.K. - 1981. Standardized measures of relative abundance based on modelling $\log$ (CPUE), and their application to Pacific Ocean perch (Sebastes alutus). J. Cons. CIEM, 39: 211-218.

Mangold, K. - 1983. Eledone moschata. In: P.R. Boyle (ed.), Cephalopod life cycles. Vol. 1, pp. 387-400. Academic Press, London.

Mangold, K., S.V. Boletzky and D. Frosch. - 1971. Reproductive biology and embryonic development of Eledone cirrhosa (Cephalopoda, Octopoda). Mar. Biol., 8: 109-117.

Papaconstantinou, C. - 1990. The spreading of Lessepsian fish migrants into the Aegean Sea (Greece). Sci. Mar., 54: 313-316.

Papaconstantinou, C. and H. Farrugio. - 2000. Fisheries in the Mediterranean. Medit. Mar. Sci. 1: 5-18.

Papaconstantinou, C. and N. Tsimenides. - 1979. Some uncommon fishes from the Aegean Sea. Cybium, 7: 3-14.

Pauly, D., V. Christensen, J. Dalsgaard, R. Froese and F. Torres Jr. 1998. Fishing down marine food webs. Science, 279: 860-863.

Punt, A.E., T.I. Walker, B.L. Taylor and F. Pribac. - 2000. Standardisation of catch and effort data in a spatially-structured shark fishery. Fish. Res., 45: 129-145.

Sinis, A.I. and A.S. Koukouras. - 1995. New information on the Aegean Sea ichthyofauna. Cybium, 19: 421-424.

Stergiou, K.I and E.D. Christou. - 1996. Modelling and forecasting annual fisheries catches: comparison of regression, univariate and multivariate time series methods. Fish. Res. 25: 105-138.

Stergiou, K.I, E.D. Christou, D. Georgopoulos, A. Zenetos and C. Souvermezoglou. - 1997a. The Hellenic Seas: physics, chemistry, biology and fisheries. Ocean. Mar. Biol. Ann. Rev., 35: 415-538.

Stergiou, K.I, E.D. Christou and G. Petrakis. - 1997b. Modelling and forecasting monthly fisheries catches: comparison of regression, univariate and multivariate time series methods. Fish. Res., 29: 55-95.

Stergiou, K.I. and M. Koulouris. - 2000. Fishing down the marine food webs in the Hellenic seas. CIESM Work. Ser., 12: 73-78.

Theocharis, A. and A. Lascaratos. - 2000. Possible causes, origin, evolution and some consequences of the Eastern Mediterranean Transient during the period 1987-1999. CIESM Work. Ser., 10: 41-44.

Tserpes, G., P. Peristeraki, G. Potamias and N. Tsimenides. - 1999. Species distribution in the southern Aegean Sea based on bottom-trawl surveys. Aquat. Liv. Res., 12: 167-175.

Tsimenides, N., G. Tserpes, A. Machias and A. Kallianiotis. 1991. Distribution of fishes on the Cretan self. J. Fish Biol., 39: 661-672. 